waves and corpuscles; spectral lines and the structure of atoms; molecular structure (and spectra); structure of solids and liquids; electrodynamics of moving media; matter and energy in the universe.

Great attention is paid to securing a clear and understandable presentation of each item of the very wide range covered, and the utmost care is taken to safeguard the interests of those who are not advanced mathematicians. The standard is stated to be that of an ordinary degree. Frequent reference is made to the preceding volumes in which attendant subjects have been developed, and hence the order here followed is somewhat unusual. But insufficient stress is laid on the interrelation of all the vast field of phenomena, and as a result little attention is directed to the efforts of contemporary study to unify the heterogeneous accumulation of facts and theories embraced by modern physics; there is, indeed, practically no discussion of the formal, abstract and philosophical bases of the science. The recent discoveries of the positron and neutron are briefly described, the Heisenberg mechanics is not attempted, and the work of Dirac is almost unmentioned, but the simplified exposition of the Schrödinger wave equation is extremely good, as are the very numerous photographs of spectra, tracks, patterns, etc.; the printing and production are of the highest order.

The student who has previously consolidated a reasonable framework of knowledge will have no difficulty in filling in from these pages such details, apart from formal mathematics and abstract theory, as his personal needs and aims dictate; the book will also provide a valuable source of reference, and the teacher and lecturer will find his requirements supplemented in a concise and accessible form.

N. M. B.

\section{The Structure of Crystals}

By Ralph W. G. Wyckoff. Supplement for 1930-1934 to the second edition. (American Chemical Society, Monograph Series No. 19A.) Pp. 240. (New York: Reinhold Publishing Corporation, 1935.) 6 dollars.

THE supplement to the second edition of Wyckoff's book on "The Structure of Crystals" records the progress made in the years 1930-34. The 367 references in the original volume to work done in the year 1930 have now been supplemented by 129 additional references to papers published in the same year, whilst subsequent years are represented as follows: 1931 (509), 1932 (503), 1933 (546), 1934 (362). These additional references occupy about one third of the text, the remaining two thirds being used for abstracts of the new data, classified (with one exception) in the same way as the main volume.

Mention may be made of structures such as the octahedral clusters of six boron atoms in $\mathrm{CaB}_{6}$, the linear FHF ions in $\left(\mathrm{NH}_{4}\right) \mathrm{HF}_{2}$ and the non-linear $\mathrm{NO}_{2}$ ions in $\mathrm{NaNO}_{2}$. The X-ray analysis of five poly. morphic forms of ammonium nitrate is recorded, the form which is stable at highest temperatures having the body-centred cubic structure of cæsium chloride, apparently because both ions are rotating and are therefore spherically symmetrical, It has also been shown that the water remaining in plaster of Paris can be nearly all removed without destroying the crystal form and without altering the diffraction pattern, leading to the conclusion that the water is present in a zeolitic condition, occupying gaps or holes in the crystal structure of the anhydrous salt.

Examples such as these may serve to show the interest and importance of the data now recorded; but the chief value of the supplement depends on the fact that it is possible to look up the data for all the diverse compounds that have been examined by this method, and to find both a summary of the essential results and references to the original papers.

\section{Infra-Red and Raman Spectra}

By Dr. G. B. B. M. Sutherland. (Methuen's Monographs on Physical Subjects.) Pp. xi +112 . (London : Methuen and Co., Ltd., 1935.) 3s. net.

ONE of the most remarkable features of present-day physics is the rapidity with which a novel phe. nomenon or effect is absorbed into the body of general doctrine, and may indeed almost immediately become a powerful tool for the investigation of intimate structural details. In the present monograph, the author has restricted himself to the study of infra-red and Raman spectra as a means to the investigation of molecular structure. The hundred and twelve pages of the book are divided between a discussion of experimental methods, of the normal vibrations of a molecule, of vibration-rotation spectra, long wave-length spectra and the correlation of infra-red and Raman spectra. An appendix gives the inter-nuclear distances and fundamental fre. quencies of some simple molecules as determined from infra-red and Raman spectra. Bibliographies are appended to the various chapters, and include a total of more than seventy references.

The volume begins at the beginning and, though definitely restricted in scope, is none the less an admirable introduction to a more general study of the subject.

A. F.

\section{Fluorescence Analysis in Ultra-Violet Light}

By J. A. Radley and Dr. Julius Grant. (Monographs on Applied Chemistry, Vol. 7.) Second edition. Pp. $\mathrm{xi}+326+29$ plates. (London : Chapman and Hall, Ltd., 1935.) 21s, net.

ONE welcomes a second edition of this very useful book after a lapse of only two years. The authors have made little change in the scope and arrangement of the information, but they have enlarged it by fifty per cent by interpolating numerous fresh instances of the ever-broadening utility of fluorescence phenomena. The publishers have also been generous in adding fourteen excellent plates descriptive of recent developments.

The present-day craving for applying new scientific methods to criminology finds response in several examples, especially stains on garments caused by various body fluids, and the detection of drugs, ete., in body fluids, which serve as valuable aids in setting out on toxicological inquiries. Under "Medical and 